\title{
Does Working Together Work? A Systematic Review of Effectiveness of Combined Endo- Perio Interactions
}

\author{
Dr.Aruna Kanaparthy ${ }^{1}$, Dr.Jayalaxmi ${ }^{2}$, Dr.Rosaiah Kanaparthy ${ }^{3}$, Dr. Nandini T. \\ Niranjan ${ }^{4}$. \\ 1. Reader, Dept of Conservative Dentistry \& Endodontics.Peoples Dental Academy, Bhopal-462037, Madhya \\ Pradesh.HIG-3, PDA Staff quarters, Peoples campus, Bhanpur, Bhopal \\ 2. Professor \& HOD,, Dept of Conservative Dentistry \& Endodontics, Peoples College of Dental Science, \\ Bhopal-462037, Madhya Pradesh.HIG-4, PCMS, Staff quarters, Peoples campus, Bhanpur. \\ 3. Professor, \& HOD. Dept of periodontics, Peoples DentalAcademy, Bhopal-462037, Madhya Pradesh \\ HIG-3, PDA Staff quarters, Peoples campus, Bhanpur, Bhopal. \\ 4. Reader, Department of Conservative Dentistry and Endodontics, Bapuji Dental College and Hospital,
} Davangere, Karnataka, India

\begin{abstract}
Absract: Pulpal infection may cause a tissue destructive process that proceeds from the apical region of a tooth toward the gingival margin. The term "retrograde periodontitis" was suggested in order to differentiate this from marginal periodontitis in which the infection spreads from the gingival margin toward the root apex. The relationship between periodontal and pulpal disease was first described by Simring and Goldberg in $1964^{1}$. Pulpodontic-periodontic syndrome has been used to define a syndrome involving inflammation or degeneration of the pulp with a periodontal pocket adjacent to the same tooth. This syndrome can be initiated by either pulpal or periodontal disease and may manifest pulpal and periodontal symptoms. In general, pulpal infection has the potential to initiate inflammatory changes in the alveolus at both apical and non-apical locations of teeth. Combined lesions are those that occur where an endodontically induced periapical lesion exists on a tooth that is also periodontally involved. All of these conditions can be placed under the general term periodontitis associated with pulpal disease.
\end{abstract}

Keywords- Endodontic, Periodontics, Pulpodontic-periodontic syndrome, Retrograde periodontitis.

\section{Introduction:}

Communication between endodontic and periodontal lesions ${ }^{2,3,4,}$ The periodontium communicates with pulp tissues through many channels or pathways. These channels may be involved in extending pulpal infections to the periodontium and vice versa.

Lateral and Accessory Canals and Foramina, ${ }^{4,5 .}$ Studies of human teeth have shown that lateral and accessory canals and foramina are present in great numbers, especially in the bifurcation and trifurcation regions of molars. Some investigators found accessory canals in molar furcations in $20 \%$ to $60 \%$ of permanent teeth and in $23 \%$ of deciduous molars examined.

In a study of 1,140 extracted adult human teeth, lateral canals were found in $27 \%$ of the teeth, and distributed at various levels of the root. The lateral canals were filled with capillaries, pulp cells, ground substance and connective tissue fibers that were continuous with the pulp tissue. In many cases the width of the accessory foramina or lateral canals was exceedingly small, permitting the presence of only small caliber vessels and their supporting stroma. In another report, $2 \%$ of the 100 teeth studied had accessory canals located within a periodontal pocket and $23 \%$ had 1 or 2 accessory canals. A fairly high prevalence of lateral canals in posterior teeth that communicate with the floor of the pulp chamber and the periodontal ligament has been reported in animals. Thus, there is ample evidence to indicate that infection of the pulp can potentially communicate with the periodontium at locations other than the apex of the tooth.

\section{Apical Foramen and Other Communications ${ }^{6,7}$}

It has been shown that when apical granulomas from necrotic pulps are extensive, the granulomatous tissue can be present along the lateral aspects of roots, which may cause extensive resorption of the alveolar crest. Other communications between the periodontium and pulp may include dentinal tubules and common vasculolymphatic drainage. Although communication between pulp and periodontium can be focused on the vascular route, many other possible avenues exist.

The following anatomical entities or pathways have also been mentioned in the literature as possible causes of periodontally derived endodontic lesions:

- Lingual grooves 


\section{- Root/tooth fractures}

- Cemental agenesis/hypoplasia,

- Root anomalies,

- Intermediate bifurcation ridges,

- Fibrinous communications and

- Trauma-induced root resorption.

\section{Effect of Pulpal Diseases on the Periodontal Tissues ${ }^{6,7 .}$}

Several experimental animal studies have assessed the effect of pulpal injury on the periodontium. In most instances, lateral canals were found to be associated with the production of the periodontal lesions and led to destruction of much of the interradicular bone and bone in bifurcations. Periodontal lesions induced by pulp diseases have also been noted in humans. In a case series of 109 teeth considered to have a hopeless periodontal prognosis, $89 \%$ of the teeth were successfully retained after endodontic therapy. These results suggest that the cases were affected by a retrograde periodontitis.

In another study, $80 \%$ of the teeth subjected to pulpal and periodontal irritants had a greater incidence of inflammatory reactions than those teeth with periodontal disease alone. Several recent retrospective clinical studies have shown a correlation between degree of marginal periodontal breakdown and presence of periapical pathology in periodontitis prone patients.

\section{Effect of periodontal inflammation on the pulp ${ }^{8}$}

Although dental plaque bacteria have the potential to induce pathologic changes in the pulp along the same pathways as an endodontic infection can affect the periodontium in the opposite direction, the effect of periodontal disease on the pulp is not as clear-cut as the effect of pulpal disease on the periodontium. It has been found that inflammatory alterations or localized necrosis of pulp tissue were adjacent to lateral canals in roots exposed by periodontal disease. However, normal pulps have also been observed in teeth with advanced periodontal disease. The initial effect of periodontal inflammation on the pulp may be degenerative. Fibrosis, calcifications, and collagen resorption have been reported in pulps of teeth with long-standing periodontal disease. Irregular or reparative dentin formation has also been found with some resorption of dentin in periodontally diseased teeth. During scaling and root planing, cementum and superficial dentin are removed from the root surface by instrumentation. In one study, chronic pulpitis with bacterial penetration of dentinal tubules was found adjacent to roots of teeth that had been scaled and root planed.

\section{Microbiology ${ }^{9}$}

Early microbiological studies that assessed the microflora associated with acute suppurative dental infections implicated streptococci or staphylococci as the causative microorganisms, but more recent studies suggest that the microbiota of an acute dentoalveolar abscess is usually polymicrobial, similar to periodontal disease. Fusobacterium, Prevotella, Porphyromonas, Peptostreptococcus, and Streptococcus are the predominant genera that have been isolated. Spirochetes also reside in infected root canals. Acute exacerbations of chronic periapical infections are frequently associated with specific anaerobes, particularly Porphyromonas gingivalis and Porphyromonas endodontalis. The microbiota in necrotic pulps is not as complex as those in deep periodontal pockets. The former is usually comprised of a limited number of bacterial species of which one or two may predominate, such as black pigmented Bacteroides.

Treatment ${ }^{2,3,4,10 \text {. }}$

\section{Initial considerations}

The prognosis of the tooth should be considered carefully. Considerations include whether there is a functional need for the tooth or if it is possible to provide an adequate root filling (i.e. negotiable canals are present). Other important considerations are whether the tooth is restorable after the lesion has been treated, and patient suitability for lengthy, costly, invasive treatment with a need for high patient motivation. If any of these factors are deemed negative extraction is the treatment of choice.

\section{For endodontic lesion:}

For primary endodontic lesions conventional endodontic therapy alone will resolve the lesion. A review 4-6 months post-operatively should show healing of the periodontal pocket and bony repair.13 Surgical endodontic therapy has been shown to be unnecessary even in the presence of large periradicular radiolucencies and periodontal abscesses. 


\section{Periodontal lesions:}

Primary periodontal lesions are treated by hygiene phase therapy in the first instance. Subsequently poor restorations and developmental grooves that are involved in the lesion are removed as these are difficult areas to treat successfully. Periodontal surgery is performed after the completion of hygiene phase therapy if deemed necessary.

\section{Alternative Treatment Modalities:}

When traditional endodontic and periodontal treatments prove insufficient to stabilize affected teeth, the clinician must consider other treatment alternatives like root amputation and hemisection.

- Root amputation procedures are a logical way to eliminate a weak, diseased root. Root amputation is considered in case of defects in maxillary molars and the existence of periodontal bone loss to the extent that periodontal therapy and maintenance will not sufficiently improve the condition.

- Bicuspidization is procedure where splitting of two rooted tooth into two separate portions (premolars).

- Hemisection is the most common method of removing a pathologically involved root. Before carrying out any such procedures the occlusal forces, restorability and the value of the remaining roots must be examined.

\section{Objective:}

The objective of this study was to evaluate longitudinal studies in effectiveness of treatment of periodontal-endodontic lesions and the influence of periodontal or endodontic procedures through a systematic review.

\section{Methodology:}

The investigation was a structured review of studies involving a longitudinal quantitative systematic review. For the selection of articles we included in prospective studies before the influence of therapy primarily in endodontics or periodontics and the efficacy of treatment of the lesion involving endodontic-periodontal disease. Thus, we used sources of cataloging electronic literature identified by MEDLINE and Cochrane Collaboration. The MEDLINE database includes international literature both medical and biomedical, produced by the National Library of Medicine - USA. The search of articles in the MEDLINE database was obtained from the PubMed website (http://www.ncbi.nlm.nih.gov/PubMed) during the years 1966 to 26 March 2011, using various combinations of keywords, as follows:

- Periodontal- endodontic lesion $(n=14)$

- $\quad$ or endodontic-periodontal lesions $(\mathrm{n}=16)$

- periodontal or endodontic lesion $(n=82)$

- endodontic or periodontal lesions $(\mathrm{n}=152)$

- or endo-perio lesion $(\mathrm{n}=3)$

- or endo-perio lesions $(\mathrm{n}=9)$

- endodontics or periodontal lesion $(n=70)$

- endodontics periodontal lesions $(\mathrm{n}=12)$

The search strategy for systematic reviews in database Cochrane Library was conducted through a search on the website of Oral Health Group.

(http://www.ohg.cochrane.org/reviews.html).

The studies were selected by two reviewers, independently who also determined the criteria for inclusion and exclusion. The search revealed 271 related articles, and of these, 59 articles were literature reviews, 31 articles were related to prospective studies, 76 studies were clinical case reports, 19 studies were conducted in animals and 25 retrospective studies. The Inclusion and exclusion criteria are represented in Table $1 \& 2$

\section{Results:}

It was observed that there was a lack of longitudinal studies that met the inclusion criteria, to perform an evidence-based analysis (Table 3.). However, the literature pointed out that successful treatment of lesions of endodontic-periodontic origin is related to the identification of the etiology, control of microbiota present, the immunological characteristics of the individual, and one of the strategies and the possible prognosis is linked to the origin of the process infection (endodontic or periodontal). Considering the clinical success of endodonticperiodontal lesions, suggesting a control of initial microbial load of oral cavity, followed by control of the endodontic microbiota, followed by the periodontal, and finally to perform the treatment. The individual's immune system actively participates in the process as managing component. 


\section{Discussion}

Concern about the treatment of periodontal-endodontic lesions resulted in a literature search to allow reflection with a view to making decisions as to its effectiveness against the influence of periodontal or endodontic procedures. Thus, several precautions were taken regarding the designing this type of study. This systematic review followed a structure recommended by the Cochrane Database of Systematic Reviews, guided by the following steps: 1) formulation of the question, 2) location and selection of studies, 3) critical evaluation of studies, 4) data collection; 5) analysis and presentation of data, 6) data interpretation; 7) improvement and updating of the review (http://www.cochrane.org).

The confirmation of the studies based on evidence has been widely appreciated as an exclusion factor in systematic reviews. ${ }^{11,12,13}$ Many decisions that guide the clinical protocols treatments have been grounded in conflicting results and often inconclusive. ${ }^{13}$ Systematic reviews or meta-analysis have been proposed to guide clinical decision making, and indicate a sedimentary response to arguments and experience. Because of studies with concordant and discordant findings, one wonders if the path more consistent with research evidence, especially in view of the enormous number of information..$^{13}$ One caution that has always should be checked in the investigations involves the adoption of clinical procedures from the extrapolation of the results, which are often not conclusive, which do not allow a clinical decision for treatment protocols in Humans. ${ }^{14}$ In this sense, Siwek et al. (2002) $)^{15}$ related levels of evidence into three categories: Level A (controlled studies randomized / meta-analysis) Level B (a non-randomized clinical study, well defined) Level C (consensus / personal opinion). It is expected that the systematic review and meta-analysis is able to determine whether a clinical procedure is valid or not. The clinical relevance questioning focuses on such research. Another essential point is linked to the respect and perception adopted in search of articles, selection of inclusion and exclusion criteria, publication bias, studies and the hierarchy of analysis criteria. Factors such as those who value the same way study also externalize their limitations ${ }^{13,16}$ analyzed the quality of published systematic reviews that related to dental interventions.

This study pooled in a search related articles 271 , of these, 59 articles were literature review, 19 articles were related to animal studies, 76 studies were case reports clinical, 31 articles were prospective studies and 25 studies were retrospective in humans. Of the 257 studies, no articles met the inclusion criteria, precluding the analysis of data based on evidence. It is prudent to note that even considering 31 prospective articles, within the keywords used in search (endodontic-periodontal periodontal or endodontic lesion, endodontic or periodontal lesions or lesion endodontic or periodontal lesions or endo-perio lesion or endo-perio lesions,Periodontal endodontics, endodontics periodontal lesion or lesions) no article met the inclusion criteria.

This study showed that quality of systematic reviews published in dentistry needs to be improved. When clinical decisions are based on systematic reviews, it is essential that this work has relevance clinically, focusing on important issues and to present a methodologically well-designed approach which is clear and reproducible in outcome.

\section{Conclusion:}

Within the limitations of the study methodology we conclude that there were no longitudinal studies that met the inclusion criteria with a view for evidence-based analysis. However, the literature pointed out that successful treatment of lesions of endodontic-periodontic origin is related to the identification of the etiology, control of microbiota present, the immunological characteristics of the individual, and one of the strategies and the possible prognosis is linked to the origin of the process infection (endodontic or periodontal). Considering the clinical success of endodontic-periodontal lesions, it is suggested that a control of initial microbial load of oral cavity, followed by control of the endodontic microbiota, followed by the periodontal and finally to perform the required treatment.

\section{References:}

[1]. Simring M, Goldberg M. The pulpal pocket approach: retrogradeperiodontitis. J Periodontol 1964; 35 : 22-48.

[2]. Cohen S, Burns RC. Pathways of the pulp, 4th Edition, 1990

[3]. Newman, Takei, Carranza, et al. Carranza's Clinical Periodontology, 10th edition, 2007.

[4]. Lindhe J, Karring T and Lang NP. Clinical Periodontology and Implantology, 4th edition, 2003.

[5]. Grossman IL. Endodontic Practice, 11th Edition, 1988.

[6]. Meng HX. Periodontic-Endodontic Lesions: Ann Periodontology 1999; 4: 84-89.

[7]. Rose LF, Mealey BL, Genco RJ, et.al. Periodontics: Medicine, Surgery and Implants, 2004

[8]. Lewis MAO, MacFarlane TW, McGowan DA. A microbiological and clinical review of the acute dentoalveolar abscess. Br J Oral Maxillofac Surg 1990; 28:359-366.

[9]. Bender IB, Seltzer S. The effect of periodontal disease on the pulp. Oral Surg Oral Med Oral Pathol 1972; 33:458-474.Walton RE, Torbinejad M. Principles and practice of endodontic, $1^{\text {st }}$ Edition, 1986.

[10]. Law A, Messer H. An evidence-based analysis of the antibacterial effectiveness of intracanal medicaments. J Endod. 2004;30(10):689-94.

[11]. Kojima K, Inamoto K, Nagamatsu K, Hara A, Nakata K, Morita I, Nakagaki H, Nakamura H. Su cess rate of endodontic treatment of teeth with vital and nonvital pulps. A meta-analysis. Oral Surg Oral Med Oral Pathol Oral Radiol Endod. 2004;97(1):95-9. 
[12]. Estrela C, Caesar OVS, Leles CR, Pimenta FC, Alencar AHG.evaluationin longitudinal studies of the effectiveness of calcium hydroxide on the Enterococcus faecalis in endodontic infections-Review Systematic. Rev Bras Odontol. 2007.

[13]. Estrela C, Marcelo VC, Sabino GA. Scientific Work. In: Estrela C. Scientific Methodology. New York: Pergamon Press, 2005. p.151-83.

[14]. Siwek J, Gourlay ML, Slawson DC, Shaughnessy AF. How to write an evidence-based clinical review article. Am Fam Physician.2002;65(2):251-8.

[15]. Glenny AM, Esposito M, Coulthard P, Worthington HV. The assessment of systematic reviews in dentistry. Eur J Oral Sci.2003;111:85-92. 